\title{
Quantifying Global Drivers of Zoonotic Bat Viruses:
}

\section{A Process-Based Perspective}

\author{
Liam Brierley, ${ }^{1,2, *}$ Maarten J. Vonhof, ${ }^{3}$ Kevin J. Olival, ${ }^{4}$ Peter Daszak, ${ }^{4}$ and Kate E. Jones ${ }^{1}$ \\ 1. Institute of Zoology, Zoological Society of London, Regent's Park, London NW1 4RY, United Kingdom, and Centre for Biodiversity and \\ Environment Research, Department of Genetics, Evolution and Environment, University College London, Gower Street, London, WC1E \\ 6BT, United Kingdom; 2. Centre for Immunity, Infection and Evolution, Institute of Evolutionary Biology, University of Edinburgh, \\ Ashworth Laboratories, King's Buildings, Charlotte Auerbach Road, Edinburgh EH9 3FL, United Kingdom; 3. Department of Biological \\ Sciences and Environmental and Sustainability Studies Program, Western Michigan University, Kalamazoo, Michigan 49008; 4. EcoHealth \\ Alliance, 460 West 34th Street, New York, New York 10001
}

Submitted January 29, 2015; Accepted July 22, 2015; Electronically published January 5, 2016

Online enhancement: appendixes, zip file. Dryad data: http://dx.doi.org/10.5061/dryad.ds2nj.

\begin{abstract}
AвSTRACT: Emerging infectious diseases (EIDs), particularly zoonoses, represent a significant threat to global health. Emergence is often driven by anthropogenic activity (e.g., travel, land use change). Although disease emergence frameworks suggest multiple steps from initial zoonotic transmission to human-to-human spread, there have been few attempts to empirically model specific steps. We create a process-based framework to separate out components of individual emergence steps. We focus on early emergence and expand the first step, zoonotic transmission, into processes of generation of pathogen richness, transmission opportunity, and establishment, each with its own hypothesized drivers. Using this structure, we build a spatial empirical model of these drivers, taking bat viruses shared with humans as a case study. We show that drivers of both viral richness (host diversity and climatic variability) and transmission opportunity (human population density, bushmeat hunting, and livestock production) are associated with virus sharing between humans and bats. We also show spatial heterogeneity between the global patterns of these two processes, suggesting that high-priority locations for pathogen discovery and surveillance in wildlife may not necessarily coincide with those for public health intervention. Finally, we offer direction for future studies of zoonotic EIDs by highlighting the importance of the processes underlying their emergence.
\end{abstract}

Keywords: emerging infectious diseases, zoonoses, hotspots, land use, viral richness, bats.

\section{Introduction}

Human emerging infectious diseases (hereafter, EIDs) are a global health priority (Morse et al. 2012). Though "emerging" is poorly defined in the literature (Funk et al. 2013), it is usually considered to involve a pathogen recently moving

* Corresponding author; e-mail: 1.brierley@sms.ed.ac.uk.

Am. Nat. 2016. Vol. 187, pp. E000-E000. (C) 2015 by The University of Chicago. 0003-0147/2016/18702-56023\$15.00. All rights reserved.

DOI: $10.1086 / 684391$ into humans for the first time, increasing in incidence and/ or geographic range, or exhibiting higher pathogenicity in humans (Morse 1995; Taylor et al. 2001). Approximately $60 \%-75 \%$ of reported human EIDs (Taylor et al. 2001; Woolhouse and Gowtage-Sequeria 2005; Jones et al. 2008) are zoonotic; therefore, a focus on the human-animal epidemiological interface is essential to understanding the emergence of the majority of EIDs.

Disease emergence depends on a series of drivers, which include socioeconomic and environmental changes (Smolinski et al. 2003; Karesh et al. 2012). Although a wide range of drivers have been traditionally proposed, these have only recently been considered from the perspective of disease emergence as a series of steps from initial zoonotic transmission to human-to-human spread. Several stepwise frameworks for EIDs have been proposed (Wolfe et al. 2007; Morse et al. 2012), with some distinguishing between predicted ecological drivers of each step (Lloyd-Smith et al. 2009; Mollentze et al. 2014). As well as varying between processes, emergence drivers also vary spatially as they often involve landscape-level changes. Therefore, environment and biogeography represent a significant determinant of EID risk (Patz et al. 2004). To better understand the ecological drivers and global patterns of emerging zoonoses, we build on these approaches as well as models of driver interactions (Plowright et al. 2008; Wood et al. 2012) to create a processbased stepwise framework (fig. 1). We then use our framework to inform an empirical spatial model of drivers of earlystage disease emergence.

Emerging zoonoses begin when pathogens in a natural host (fig. 1A, step 1) spill over (i.e., transmit to a novel host) into human individuals (fig. $1 A$, step 2). This crossspecies transmission is dependent on physical opportunity, for example, occupation of the same environment or fre- 


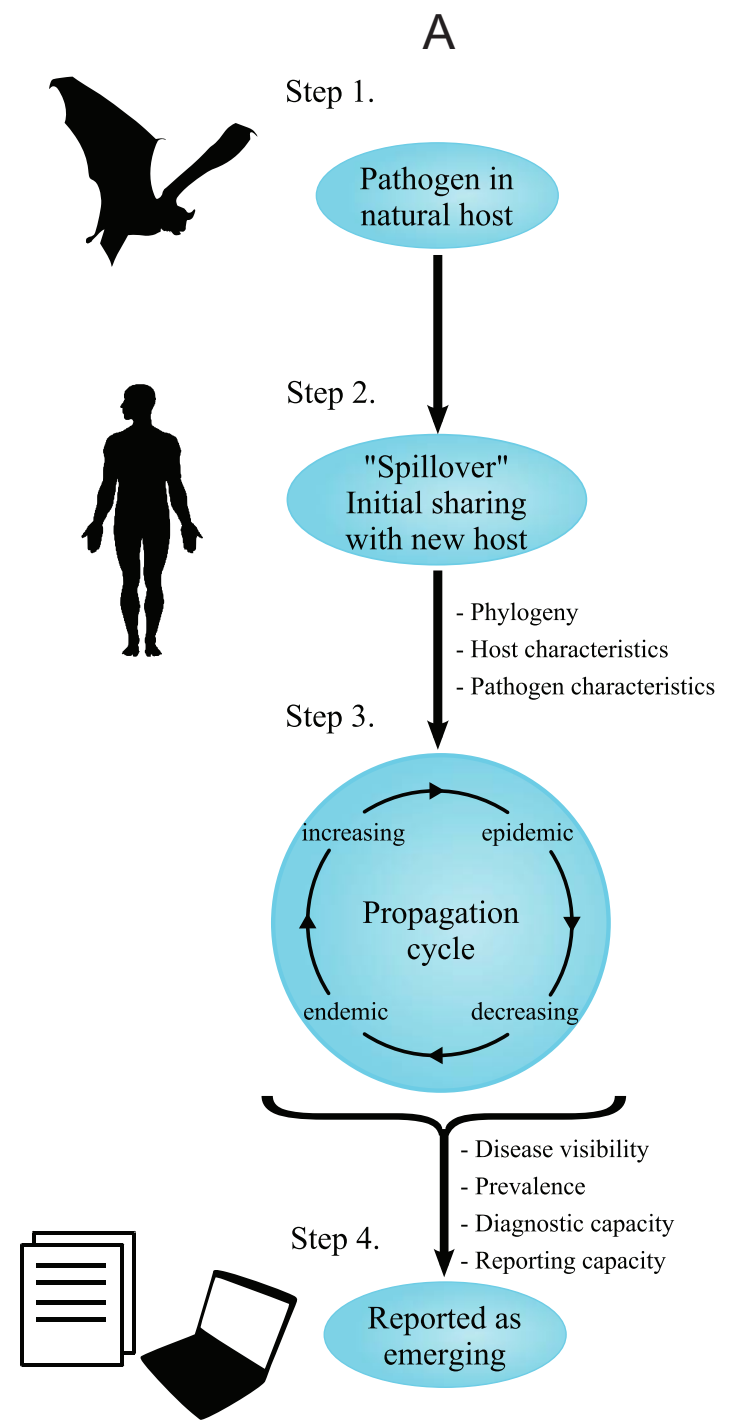

B

Step 1.

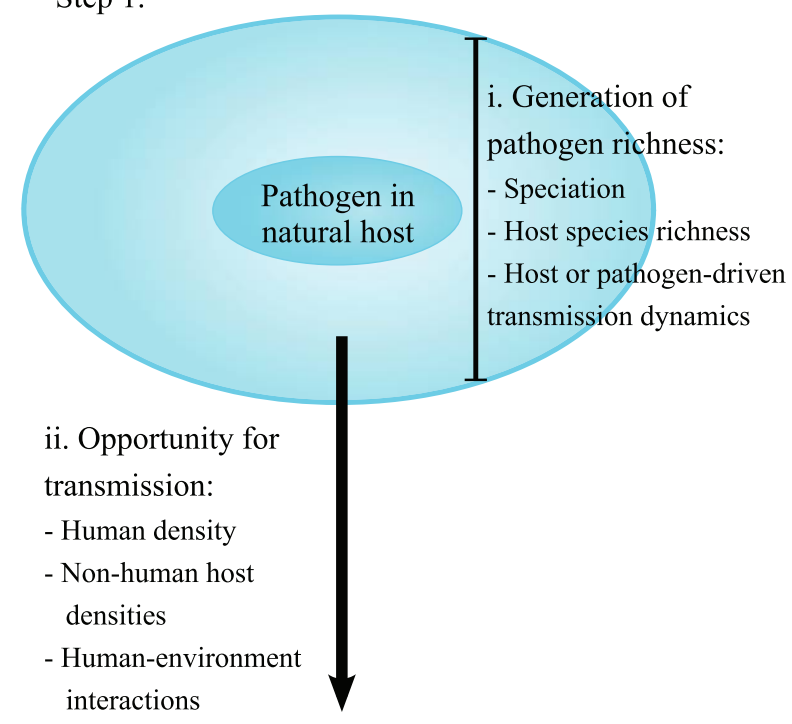

iii. Establishment

within individual:

- Phylogenetic relatedness

- Infection dynamics e.g.,

immunity

- Healthcare quality

Step 2.

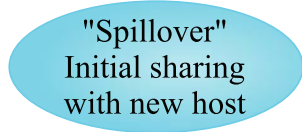

Figure 1: Process-based framework of zoonotic pathogen emergence for the whole pathway $(A)$ and between steps 1 and $2(B)$, expanded with necessary processes for spillover, labeled i, ii, and iii. Processes are verbally described to avoid conflicting terminology; for example, "opportunity for transmission" has been differentially referred to as "contact" in emerging disease literature and "exposure" in host-parasite literature. Drivers are listed next to the arrows between steps.

quency of direct or indirect contact (Pulliam 2008; Pedersen and Davies 2009; fig. 1B). Both successful cross-species transmission and transmission between individuals of the new host species (fig. $1 A$, steps 2 and 3 ) are dependent on how phylogenetically related the novel and natural host are (Wolfe et al. 2000; Streicker et al. 2010; Cooper et al. 2012), as immunological and molecular environments (e.g., cell receptors) are more similar between closely related hosts (Morse et al. 2012). Propagation between individuals of a new host species can exhibit complex epidemiological dynamics with variable outcomes, for example, cyclic increases and decreases but also endemicity or extinction (Anderson et al. 1986). Given the definitions outlined earlier, a zoonotic pathogen may be reported as emerging or reemerging at any point (fig. $1 A$, step 4 ), subject to surveillance capacity and disease characteristics.

Few studies to date have attempted to empirically model specific steps, and those existing models tend to focus toward the later stages. For example, the phylogenetic determinant of reaching step 3 (fig. $1 A$ ) has been modeled across a number of taxonomic groups (Pedersen and Davies 2009; Streicker et al. 2010; Cooper et al. 2012), and for zoonoses, 
step 4 (fig. 1A) has been spatially modeled in the form of reported emergence events (Jones et al. 2008). Here, we focus on empirically modeling the drivers of cross-species transmission, or spillover (fig. 1, transition from steps 1 to 2), an area where current epidemiological models are noted to be deficient (Lloyd-Smith et al. 2009). In addition to empirical modeling, addressing the spatial ecology surrounding drivers has been urgently advised (Patz et al. 2004). Characterizing spatial variation in zoonosis risk and its causes would offer valuable predictions of potential future disease emergence locations (Morse et al. 2012) and insights into biogeographic mechanisms of human-wildlife interaction. Therefore, we conduct our empirical model in a spatial context to identify priority high-risk regions (termed hotspots) and to further understand the determinants of these patterns.

As a study system, we model zoonotic viruses from bats (order: Chiroptera), hereafter, defined as viruses shared between bats and humans. Bats make an excellent group for studying global anthropogenic drivers, as most known batorigin zoonoses have emerged recently compared to other zoonotic hosts, which likely reflects increasing intensification of human activity and subsequent bat-human interactions and/or increasing surveillance (Halpin et al. 2007; Plowright et al. 2011; Pulliam et al. 2012). In restricting our focus to one host group, we minimize variation between host species in their phylogenetic distance from humans. We focus on viruses, as bats are known to host a large number of viruses spanning 15 viral families (Calisher et al. 2006; Olival et al. 2012; Luis et al. 2013) and are the suspected natural hosts of several high-impact human viruses (Chua et al. 2003; Li et al. 2005; Leroy et al. 2009; Memish et al. 2013). Although comparative analyses of bat viral richness and sharing with humans have been carried out (Turmelle and Olival 2009; Luis et al. 2013), we are not aware of any comparative analyses that examine ecological drivers of such patterns in a spatial framework.

To investigate drivers of zoonotic bat viruses with respect to the emergence pathway, we expand the transition between steps 1 and 2 and designate three necessary generalized processes: (i) generation of pathogen richness (hereafter, viral richness), (ii) opportunity for transmission, and (iii) establishment within an individual (fig. $1 B$ ). Based on a literature review of ecological dynamics of disease emergence, we identify spatial drivers of each process and present those most often hypothesized or cited as appropriate for bat pathogens (fig. $1 B$ ). Subsequently, we use this expansion to shape our choice of predictor variables in spatially modeling associations between drivers and virus sharing between bats and humans. As there is insufficient spatially formatted data to model specific drivers of process (iii) for bat viruses, we focus on the first two processes. Greater viral richness is anticipated to increase risk of a successful zoonotic transmission event (Wolfe et al. 2000). Viral richness is influenced by a range of factors, including evolutionary dynamics, diversity of potential hosts, and host-parasite interactions (fig. $1 B$ ). For example, global viral richness patterns vary with wildlife host richness (Jones et al. 2008; Dunn et al. 2010) and greater seasonal variability in temperature and precipitation (Guernier et al. 2004), a proxy for niche diversity or environmentally dependent transmission routes. Viral richness of individual bat species is also strongly associated with richness of sympatric bat species (Luis et al. 2013). We therefore hypothesize that bat species richness and climatic variability will correlate with viral cross-species transmission to humans.

Opportunity for transmission between natural and potential hosts will also increase cross-species transmission risk. For example, human population density is a strong predictor of zoonotic disease emergence globally (Jones et al. 2008), as high density may represent greater human-wildlife contact opportunities through population size alone or as a proxy for various forms of human activity, for example, urban expansion. Other human-environment interactions have also been suggested as drivers, for example, periurban and agricultural land use change has been linked to the emergence of Hendra and Nipah viruses from bats through domestic animals (Plowright et al. 2011; Daszak et al. 2012; Pulliam et al. 2012). Bats are also widely consumed as bushmeat in regions where human populations are expanding, which is a known risk factor for bat-borne zoonoses in Central and West Africa (Leroy et al. 2009; Kamins et al. 2011) and throughout Asia (Li et al. 2005; Mickleburgh et al. 2009). For bat-borne zoonoses, we therefore hypothesize opportunities for transmission will increase with human population density and in regions where anthropogenic land use change and bushmeat activity bring humans into contact with bats.

Using host-virus association data for bats and humans, we create proxy spatial distributions for human-shared bat viruses. We then use spatial regression to show that this virus sharing is positively associated with drivers of both viral richness (greater diversity of bat hosts and climatic variability) and opportunity for transmission (greater human population density, livestock production, and bushmeat hunting) and that the global risk patterns from these drivers differ between processes.

\section{Material and Methods}

\section{Human-Shared Bat Virus Data Set}

Data on viral infections of bats were collected from the literature from sources published between 1900 and 2010. Sources were identified systematically using the search term "bat" combined with different pathogen terms including "parasite," "pathogen," "virus," and "viral” in several jour- 
nal indexes (ISI Web of Science, BIOSIS, Google, Google Scholar) and via recursive follow-up tracing of the literature cited by each source. Data from experimental infections and serological diagnoses were excluded as serological crossreactivity can lead to virus misidentification. Data was also supplemented using entries from a recently published data set of bat virus sequences (Chen et al. 2014). Bat species synonyms were standardized using the taxonomy of Wilson and Reeder (2005); we merged synonyms and excluded any species without a matching reference. Virus names were standardized in the same way using the International Committee on Taxonomy of Viruses 9th Report (King et al. 2011). No assumptions were made on transmission method, directionality, or whether bat species maintained infection as a natural reservoir. We then calculated the number of viruses shared with humans, defined as the virus being recorded as human infective in either the UniProt virus taxonomy database (Apweiler et al. 2004) or a recent catalog of human RNA viruses (Woolhouse et al. 2013). Using this definition, our data also includes viruses in later propagation (fig. 1A, steps 3 and 4), though we assume later steps cannot be reached without having transitioned through step 1 to step 2 (fig. $1 B$ ) and therefore assume all viruses in our data set have followed this process. These data spanned 33 zoonotic viruses from 14 genera and nine families and 148 bat species from 453 literature sources (table S1; tables S1, B1 available online).

We created proxy geographic ranges for each virus by fusing together the range maps of all known bat host species (Fritz and Purvis 2010), assuming viruses were present throughout the entire range of their bat hosts as an upper-bound estimate. However, we expect this assumption to be reasonable mathematically, as previous parasite distribution models have shown robustness to this approximation (Harris and Dunn 2010; Cooper et al. 2012), and biologically, as many bat species are theoretically less restricted in range and movement than terrestrial species, giving potential for population mixing and virus transmission (Epstein et al. 2009). Projected shapefiles for each proxy virus range are available in the Dryad Digital Repository: http://dx.doi.org /10.5061/dryad.ds2nj (Brierley et al. 2016). Spatial grids were overlaid, and presence/absence was calculated to give total human-shared viruses of each cell. We used a one-decimaldegree resolution as most of our global predictor variables were at this native resolution (unless stated otherwise) and downscaling would not have improved the accuracy of our models. Shared virus counts were square-root transformed to normalize prior to regression modeling.

\section{Driver Data Sets}

We assembled spatially explicit sets of data on different drivers of viral richness and opportunity for transmission (fig. $1 B$, i and ii). These were as follows: (1) mean monthly temperature range $\left({ }^{\circ} \mathrm{C}\right) ;(2)$ annual temperature range (max. temperature of warmest month - min. temperature of coldest month; ${ }^{\circ} \mathrm{C}$ ); (3) annual rainfall range (max. rainfall of wettest month - min. rainfall of driest month; mm); (4) bat species richness, created by calculating presence/absence grids from range maps of 1,079 bat species (Fritz and Purvis 2010); (5) human population density (persons $/ \mathrm{km}^{2}$ ) in 2005 from Gridded Population of the World, version 3 (Balk and Yetman 2005); counts of (6) buffalo, (7) cattle, (8) goats, (9) poultry, (10) sheep, and (11) pigs from the Food and Agriculture Organization's Gridded Livestock of the World, 2007 (Wint and Robinson 2007); (12) cropland/pasture cover, calculated by summing the proportion of land used per grid cell for cropland and pasture in 2000, obtained from Ramankutty (2010); and (13) bat bushmeat activity, specified as 1 or 0 for countries where significant hunting and consumption of bats occurs or does not occur, respectively, following Mickleburgh et al. (2009). Data on (1)-(3) were obtained from the BIOCLIM 1950-2000 data set (Hijmans et al. 2005) and resampled up from $10^{\prime}$ to $1^{\circ}$ resolution using bilinear interpolation in ArcGIS v9.2 (ESRI 2006). Data on (6)-(11) were resampled from $1-\mathrm{km}$ cells to $1^{\circ}$ resolution by overlaying a grid and recalculating using zonal statistics in ArcMap v9.2 (ESRI 2006). Bushmeat data was converted to grid-cell format by matching grid cells to countries as defined by Sandvik (2008).

Reporting effort for infectious diseases is likely to correlate with health-care quality and availability for humans (Jones et al. 2008; Chan et al. 2010) and surveillance resources for wildlife (Hopkins and Nunn 2007). As unequal reporting effort could introduce bias into our models, we controlled for this by including the following additional variables: (14) total number of authors per country from the Journal of Infectious Diseases from 1973 onward, following methods from Jones et al. (2008); (15) total number of authors per country from bat research papers from 1973 onward; and (16) country-level gross domestic product (GDP) per capita from 2000, in US dollars (based on 2005 exchange rates) from the World Bank Development Indicators data set (World Bank Group 2010). Bat research papers were obtained by searching Web of Science v5.0 for the binomial names of the 148 bat species in this study and their synonyms (Wilson and Reeder 2005). Data on (14)-(16) were converted to grid-cell format by matching grid cells to countries as before.

To detect any redundancy among driver variables, correlations between them were examined. Based on the correlation matrix (fig. A1, available online), annual temperature range (2); counts of buffalo, cattle, goats, and poultry (6-9); and total number of authors per country from the Journal of Infectious Diseases (14) were excluded. All remaining continuous variables were log transformed before 
modeling to normalize, except mean monthly temperature range (1), which showed approximate normality under a quantile-quantile plot, and proportion cropland/pasture cover (12), which was arcsine transformed. Transformations did not introduce further variable redundancy. Approximately $40 \%$ of terrestrial grid cells had no viruses or were missing covariate data and were thus excluded, leaving 10,124 cells.

\section{Model Construction}

Our gridded human-shared bat viruses showed strong spatial autocorrelation (Moran's $I=0.893, P=.001,999$ simulations). To account for this, we constructed a spatially explicit linear model using a conditional autoregressive (CAR) specification. The CAR specification corrects for both autocorrelation in response and explanatory variables by weighting expected values of outcome variables toward those of neighboring cells and weighting model coefficients based on residual fits of neighbors (Besag 1974; Dormann et al. 2007). The distance within which cells were considered neighbors was set to $645 \mathrm{~km}$ to ensure no cells had zero neighbors. Stepwise removal of terms that did not significantly improve model fit was then carried out using likelihood ratio tests. Data manipulation and modeling were conducted in $\mathrm{R}$ 3.1.1 (R Development Core Team 2011), and spatial regression models were carried out using the package spdep, version 0.5-77 (Bivand and Piras 2015), and scripts modified from Dormann et al. (2007).

\section{Results}

\section{Patterns of Bat-Human Virus Sharing}

All 33 human-shared bat viruses within our data set were RNA viruses, the most common genera being Flavivirus $(n=8)$ and Lyssavirus ( $n=7$; table S1). The most geographically widespread viruses were European bat lyssaviruses 1 and 2 (Rhabdoviridae: Lyssavirus), found in 16 and 8 bat species covering 6,818 and 6,295 grid cells, respectively, and St. Louis encephalitis virus and Japanese encephalitis virus (Flaviviridae: Flavivirus), found in 3 and 12 bat species across 5,688 and 5,553 cells, respectively (table S1). Our proxy distributions showed hotspots of virus sharing primarily in sub-Saharan Africa, as well as in South and East Asia, Southern Europe, and Central America (fig. 2A). The mean number of shared viruses per cell was 6.57 , and the maximum of 16 viruses per cell was found across West Africa and localized parts of East Africa (fig. 2A). Both familylevel diversity and number of species of shared viruses was highest in the Eastern Mediterranean World Health Organization region and lowest in the Americas (fig. $2 A, 2 B$ ). Families were mostly well represented globally, though proxy potential ranges of Filoviridae shared with humans were restricted to Eurasia and Africa (fig. 2B).

\section{Drivers of Bat-Human Virus Sharing}

Correcting for study and reporting biases, the final model of virus sharing contained both drivers of viral richness and opportunity for transmission (table 1). Monthly temperature range, sheep stocks, and agricultural land cover (cropland/ pasture) did not significantly improve model fit and were excluded from the final model (table B1). The fitted spatial coefficient, $\lambda$, suggested a high degree of autocorrelation in both virus sharing and model covariates $(\lambda=0.981)$. Correlation between fitted model values and observed values was high $(\rho=0.954)$ and, when decomposed, showed a relatively high contribution to model fit from spatial autocorrelation $(\rho=0.728)$ compared to contribution from model covariates $(\rho=0.763)$. For drivers of viral richness, areas with higher bat species richness and annual rainfall ranges had more bat-human shared viruses (table 1). Drivers of opportunity for transmission were marginally less influential than drivers of viral richness (table 1), though greater bathuman virus sharing was associated with higher human population densities, bushmeat hunting and consumption, and higher pig stocks. Bias corrections of country-level bat publications and GDP per capita both exhibited an unexpected negative relationship with bat-human virus sharing.

Spatially mapped values from the final CAR model showed differences in hotspots when comparing the overall final model and drivers of viral richness and drivers of opportunity for contact separately (fig. 3A-3C). Considering drivers of viral richness alone, risk of bat-human virus sharing showed prominent hotspots in Central/South America, sub-Saharan Africa, and parts of Southeast Asia (fig. 3B), whereas risk associated with drivers of opportunity for transmission was primarily concentrated in South and East Asia (fig. 3C). For both overall and separately plotted drivers, the Tropics tended to show greater bat-human virus sharing than temperate zones, though in some cases Eurasia presented a relatively high risk (fig. $3 A, 3 C$ ).

\section{Discussion \\ Patterns of Bat-Human Virus Sharing}

Our proxy ranges showed virus-sharing hotspots in subSaharan Africa and Southeast Asia, areas from which batorigin zoonotic viruses have recently emerged, for example, several filoviruses in Africa (Towner et al. 2007; Leroy et al. 2009) and Nipah virus and SARS coronavirus in Southeast Asia (Chua et al. 2003; Guan et al. 2003). Notably, the highest-risk hotspot identified in West Africa has recently ex- 

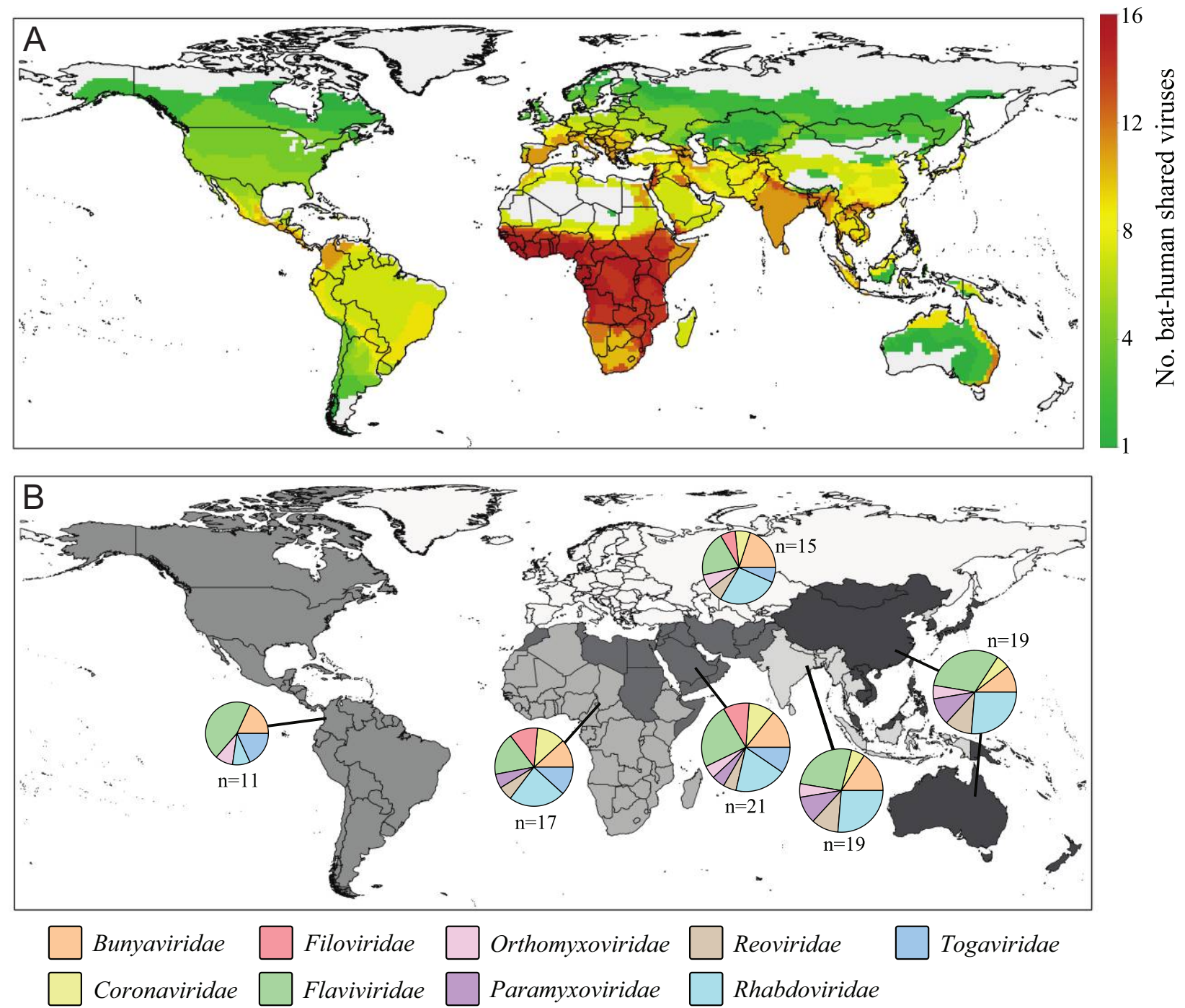

Figure 2: Distributions of bat-human virus sharing showing numbers of bat-human shared viruses at $1^{\circ}$ grid resolution $(A)$ - color represents a linear scale from 1 (green) to 16 (red) - and composition of viruses by family within the six World Health Organization World Regions $(B)$, where the size of the charts is proportional to the number of viruses. Shading denotes region (from lightest to darkest: Europe, South Asia, Africa, Americas, Eastern Mediterranean, Western Pacific), while colors denote viral family (see key).

perienced the largest-scale outbreak of Ebola virus disease yet seen, occurring outside the previously proposed endemic range (Bausch and Schwarz 2014), and the African hotspot patterns we show also coincide with recent predictive maps for the ecological niche of zoonotic ebolavirus transmission (Pigott et al. 2014). Our proxy filovirus ranges also covered parts of Eurasia (fig. $2 B$ ), though zoonotic filovirus transmission has not been reported within this region to date. However, Lloviu virus, a novel filovirus with unknown zoonotic potential, has recently been discovered in Southern European bats (Negredo et al. 2011). Our patterns also broadly parallel previously modeled hotspots of disease emergence in humans (Jones et al. 2008) and pathogen sharing between humans and primates (Pedersen and Davies 2009).

\section{Drivers of Bat-Human Virus Sharing}

Both drivers influencing viral richness and drivers influencing opportunity for transmission were significantly associated with bat-human virus sharing. Bat species richness may correlate with viral richness through host-virus codivergence. Although not yet systematically demonstrated, codivergence has been hypothesized to explain phylogeo- 
Table 1: Drivers of bat-human virus sharing within the final spatial conditional autoregressive model, separated by their associated type of process

\begin{tabular}{llcc}
\hline Covariate & Driver type & $Z$ statistic & Coefficient \\
\hline (intercept) & $\ldots$ & 23.81 & $1.66(1.51,1.82)^{* * *}$ \\
$\log ($ Annual rainfall range) & Richness & 7.55 & $.0529(.0375, .0683)^{* * *}$ \\
$\log ($ Bat species richness) & Richness & 53.18 & $.348(.334, .363)^{* *}$ \\
$\log ($ Human density) & Transmission & 12.41 & $.0180(.0148, .0212)^{* * *}$ \\
$\log ($ Pig stocks) & Transmission & 4.48 & $.00564(.00287, .00841)^{* * *}$ \\
Bushmeat activity & Transmission & 4.06 & $.0582(.0266, .0897)^{* *}$ \\
$\log ($ Bat publication authors $)$ & Bias & -6.54 & $-.0188(-.0252,-.0125)^{* * *}$ \\
$\log ($ GDP per capita) & Bias & -3.16 & $-.0228(-.0387,-.0069)^{*}$ \\
\hline
\end{tabular}

Note: "Richness" denotes drivers of viral richness. "Transmission" denotes drivers of opportunity for transmission. "Bias" denotes bias corrections. "Coefficient" denotes model regression slope (95\% confidence intervals in parentheses). GDP = gross domestic product.

${ }^{*} P<.01$.

${ }^{* * *} P<.001$.

graphic bat virus patterns in multiple viral families (Halpin et al. 2007). Additionally, bat species richness likely captures variation from cross-species transmission between bats, as range overlap with heterospecific bat species is a particularly strong predictor of zoonotic viral richness (Luis et al. 2013). Wider mammal species richness is also known to predict zoonotic emergence risk across all pathogen types (Jones et al. 2008). We also found greater climatic ranges (in the form of rainfall) to correlate with an area's number of shared viruses. Climatic variability has been shown to predict latitudinal gradients in viral diversity in humans (Guernier et al. 2004) and may facilitate greater virus (as well as bat and alternative host) speciation through habitat diversity or seasonality, providing niche diversity.

Anthropogenic drivers of opportunity for transmission (human population density, pig stocks, bat bushmeat activity) also predicted bat-human virus sharing. As well as simply facilitating invasion and persistence of infection, increasing human population densities can also introduce mechanistic transmission routes; for example, urbanization creates habitats for peridomestic bat species, bringing them into closer proximity to humans (Plowright et al. 2011). Human density is also the strongest anthropogenic predictor of reported zoonotic emergence events (fig. 1, stage 4; Jones et al. 2008), suggesting some drivers may influence both early and late steps in the emergence pathway. Though pig stocks were associated with virus sharing, sheep stocks were not present in the final model, which could indicate specificity in how these livestock systems ecologically interact with wild hosts - for example, Nipah virus emergence has been specifically traced to shared feeding on fruits between bats and pigs (Chua et al. 2003). Pig stocks also showed moderate covariance with human density and other livestock types (fig. A1) and may have alternatively acted as a proxy for certain agricultural or nonagricultural human activities, though we report no association between agricultural land use and virus sharing. Domestic hosts are frequently implicated in zoonotic spillover (Daszak et al. 2000), particularly for certain bat-borne viruses, for example, henipaviruses (Plowright et al. 2011; Pulliam et al. 2012), and may involve amplification of viral load or particles per transmission. Global data was not available for other domestic animals of interest, for example, dromedary camels, known hosts of zoonotic MERS coronavirus (Reusken et al. 2013). However, sheep and pigs appeared representative for common livestock groups (fig. A1). Although bat bushmeat hunting was globally associated with virus sharing, this should be interpreted cautiously, as the majority of bat consumption occurs in the Paleotropics (Mickleburgh et al. 2009). Bat exploitation still represents a critical priority in these areas as the most likely emergence route of certain zoonoses, for example, SARS coronavirus (Li et al. 2005).

Contrary to expectation, our study bias measure and GDP per capita negatively predicted virus sharing. Authorship may not reflect areas where study or sampling was carried out, and higher socioeconomic status may have predominantly represented prevention and control efforts of zoonoses rather than reporting (Dunn et al. 2010). Reporting disparities may be reduced as surveillance improves within developing regions (Chan et al. 2010). If the direction of this effect is genuine, however, it would indicate a concerning mismatch between high-risk areas and wellstudied areas, a pattern visible for primate zoonoses (Hopkins and Nunn 2007; Pedersen and Davies 2009).

More generally, this work establishes empirical support that anthropogenic activity and demography is associated with early-stage cross-species pathogen transmission. Although this has long been advocated through case studies of emerging diseases (Patz et al. 2004), few studies have quantified the relative impact of such drivers comparatively across multiple pathogens (Jones et al. 2008). Our models stress that, beyond basic spatial gradients of pathogen and 
A
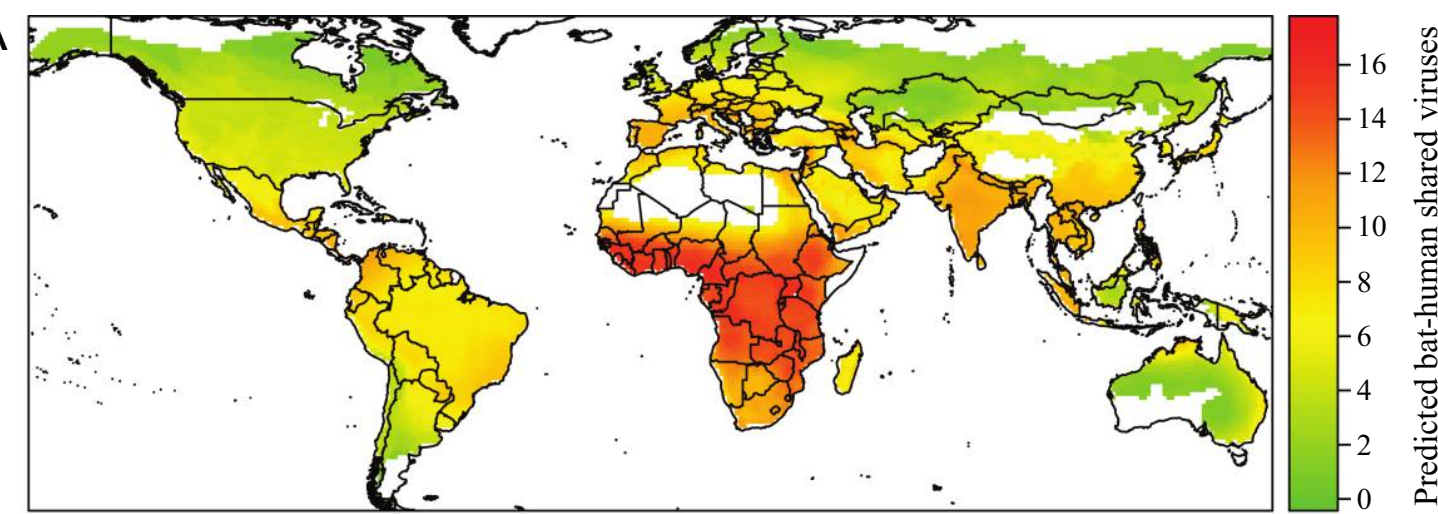

$\mathrm{B}$
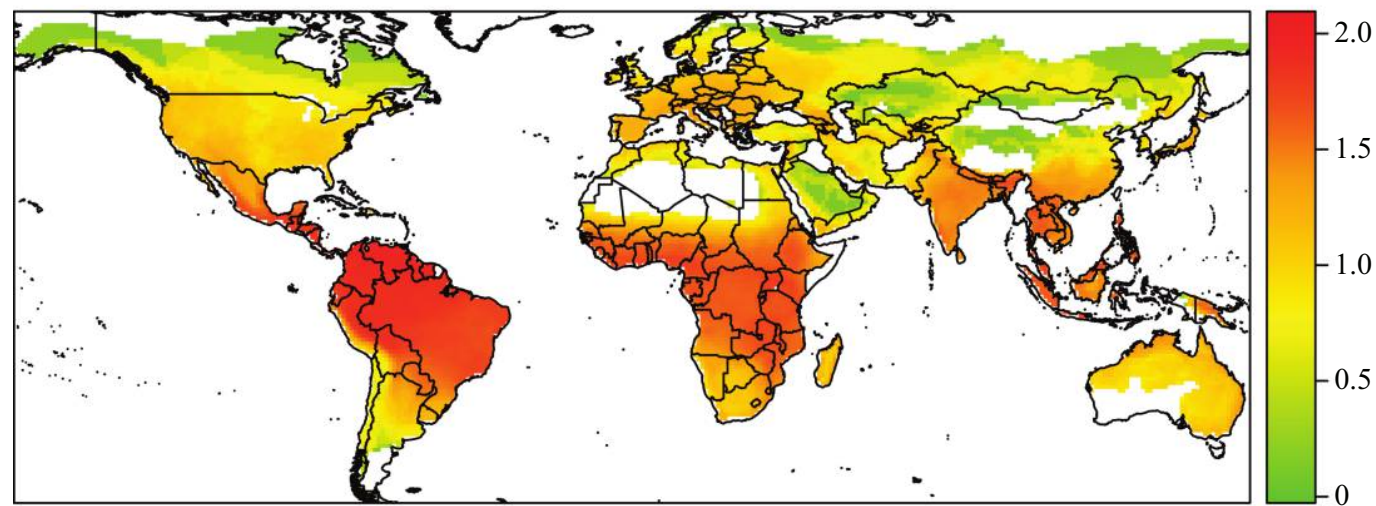

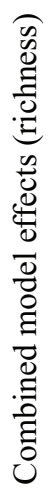
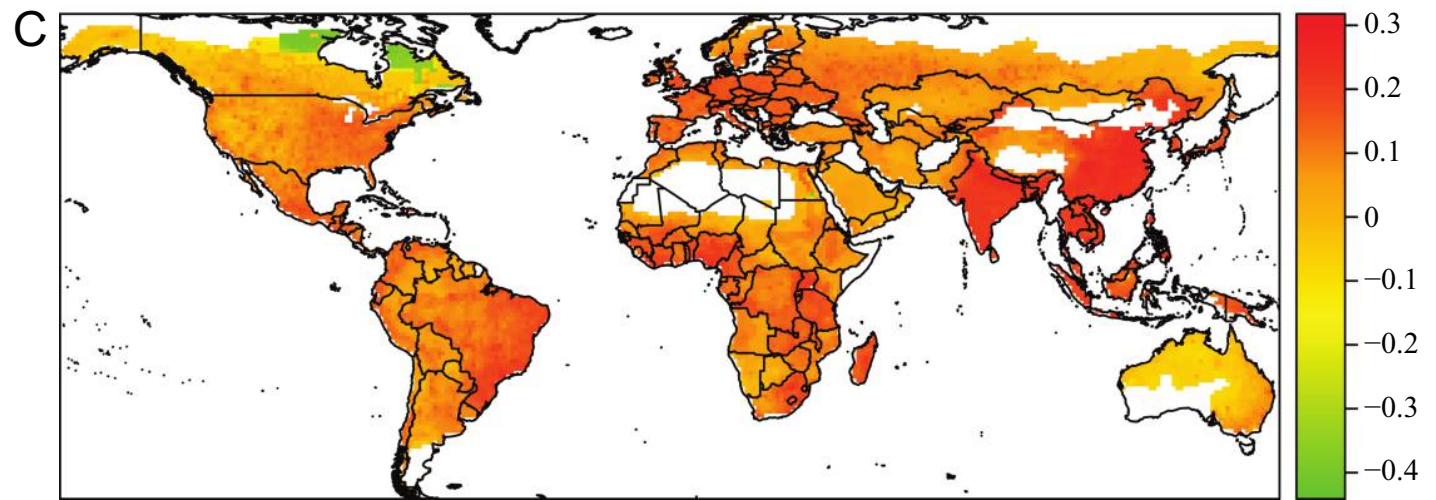

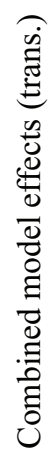

Figure 3: Spatial patterns from the final conditional autoregressive model of bat-human shared viruses at $1^{\circ}$ grid resolution for total predicted values of bat-human shared viruses $(A)$, combined effects of drivers of viral richness (richness; $B$ ), and combined effects of drivers of opportunity for transmission (trans.; $C$ ). Note that $B$ and $C$ are effects from modeling the square-root-transformed number of shared viruses, calculated mathematically, and not corrected for autocorrelation, so values have no direct interpretation. Color represents a linear scale from lower (green) to higher (red) values. Color keys do not retain the same scale between plots in order to highlight geographic differences in driver importance.

host diversity, anthropogenic activity is a significant determinant of the global distribution of emerging diseases. This supports the recent One Health perspective that human health is inherently connected with wild environments, not only through the high fraction of our pathogens that originate from wild zoonotic transmission but also through complex, expanding human-environment interfaces that facili- tate opportunity for such transmission (Daszak et al. 2000; Karesh et al. 2012).

\section{Model Assumptions}

Our empirical findings are based on several assumptions. We modeled predictors of virus species known to infect 
both bats and humans, though currently available data precludes us from inferring whether zoonotic transmission truly occurs. Bats and humans could acquire infection with viruses within our data set independently from common sources, such as environmental routes (e.g., rotavirus A), insect vectors (e.g., yellow fever virus), or contact with other mammals (e.g., Hantaan virus). Our host data was based on evidence of infection, though species are unlikely to be equally competent, with some potentially being incidental, dead-end hosts that cannot maintain infection or act as a reservoir at the population level. Reservoir status of hosts often requires substantial investment to determine (Viana et al. 2014), especially for bats, which are poorly sampled, giving very incomplete coverage of current data (Anthony et al. 2013). We model viruses at the species level, within which there may be substantial differentiation into strains. Strains may have distinct host ranges, and those known in bats may not necessarily be those infecting humans. For example, the influenza A virus H17 subtype has, to date, been found only in bats (Tong et al. 2012). Constructing viral phylogenies could potentially help to uncover directionality of bat-to-human transmission events, although sequence data is again deficient for most bat viruses. Although these caveats may overestimate zoonosis risk based on our host ranges, we posit this bias will be largely independent of geography and should not affect the comparative spatial hotspot patterns we find.

Second, we assumed virus distributions followed the combined entire distributions of their known bat hosts, equivalent to mean-field population mixing, a reasonable approximation for hosts with less restricted movement and dispersal (Webb et al. 2007). Current sampling records are consistent with this assumption for certain viruses, for example, henipaviruses (Rahman et al. 2010; Daszak et al. 2012); however, we acknowledge this will not hold true for the highly structured populations of many bat species (Rossiter et al. 2000; Miller-Butterworth et al. 2003) and, consequently, their viruses, which is likely to introduce uncertainty within our empirical model. However, using complete host ranges has been shown to be a good approximation compared to more conservative range measures when calculating spatial parasite richness for terrestrial groups (primates: Cooper et al. 2012; North American carnivores: Harris and Dunn 2010). Improvements to both certainty of bats as sources of viral zoonoses and their distribution will likely be improved by systematic, georeferenced sampling efforts in future.

As with any other macroecological analysis, our conclusions should also be interpreted in the face of potential model biases and restricted explanatory power. We aggregated covariate data sets over different geographic scales with different accuracy, as we selected the most accurate data available with global coverage, which may be improved on.
We were limited to country-level data on bushmeat hunting and GDP per capita; however, bat bushmeat hunting and associated economic pressures can be highly localized within countries (Kamins et al. 2011). Higher-resolution data would be able to better predict patterns in virus-sharing risk within countries and provide greater explanatory power.

Additionally, our model conclusions and separated spatial hotspots assume that our drivers are truly representative of the underlying processes (fig. 1) and may be subject to confounding by unmeasured relationships. For example, human population density could represent a variety of factors known to influence opportunity for transmission (e.g., travel and connectivity; Wolfe et al. 2000) or other processes such as generation of viral richness (e.g., urbanization-associated bat population shifts; Plowright et al. 2011). The multitude of determinants means that successful zoonotic transmission is ultimately a highly stochastic event, and our results should be interpreted as broad ecological associations rather than suggesting specific causal mechanisms. For further causal inference or exploration of driver interactions, our conceptual framework may be expanded into a larger causal network (Plowright et al. 2008), which can similarly act as a foundation for empirical models for testing hypotheses surrounding disease emergence.

\section{Wider Applications}

Despite the limitations outlined, large-scale predictive models can offer useful inference in disease ecology in predicting likely regions and routes of future cross-species transmission (Morse et al. 2012). Our model could be extended to identify or quantify populations at risk, as has been recently conducted for ebolaviruses (Pigott et al. 2014). The set of drivers our model highlights as influential could also direct follow-up studies in a more localized or experimental setting, to better understand their dynamics (Plowright et al. 2008).

Our approach is translatable to a range of systems. Framing spatial empirical models around conceptual frameworks (fig. 1) could offer scope for understanding and predicting emergence of wildlife infections with anthropogenic drivers (Daszak et al. 2000); for example, the amphibian chytrid fungus, Batrachochytrium dendrobatidis, is thought to be spread by global amphibian trade networks (Farrer et al. 2011). Our framework also parallels biological invasion models (Blackburn et al. 2011) by identifying the processes involved in species moving from a natural to a novel context. Stratifying empirical predictors according to the processes they drive could be valuable for invasive species management, where precision in targeting invasion pathways is critical (Hulme et al. 2008).

In separating drivers of viral richness from drivers of opportunity for transmission, our spatial model showed dis- 
parity between the hotspots for each (fig. 3), which could be used to better target public health programs (Daszak et al. 2000; Daszak 2009; Morse et al. 2012). Interventions such as bushmeat regulation and reducing bat-human or batlivestock contact (Halpin et al. 2007; Nahar et al. 2010) may be particularly effective in hotspots of opportunity for transmission (fig. 3C). However, surveillance may be best placed in hotspots of viral richness (fig. 3B), and targeted surveillance initiatives like the PREDICT program of the United States Agency for International Development (USAID 2009; Morse et al. 2012) and associated sequencing efforts (Anthony et al. 2013) will provide more complete knowledge of the bat virome, improving future empirical analyses and risk assessments. Although traditionally neglected, attention toward zoonoses from bats is growing. Bats are hypothesized to be unique as viral hosts (Olival et al. 2012), and comparative analysis has shown that bats host more zoonotic viruses per species than rodents (Luis et al. 2013). Given the pressing associations between human drivers and virus sharing, we assert that bats deserve substantial allocation of surveillance and scientific resources.

\section{Conclusion}

We offer a process-based empirical approach to further inference in studies of pathogen emergence by demonstrating the distinct underlying drivers of different processes and their distinct spatial mechanisms. Our framework and model unite ecology, epidemiology, and public health in line with recent One Health perspectives. Global risk patterns linking anthropogenic drivers and zoonotic viruses suggest that as human populations continue to expand into pristine and potentially virus-rich habitat, particularly in tropical hotspots, the threat of zoonoses will increase. Reducing opportunities for transmission, including reducing hunting pressure and disturbance of bat populations, can both mitigate zoonosis risk and provide a strong impetus for conservation programs that specifically reduce anthropogenic activity in regions of high biodiversity.

\section{Acknowledgments}

This work was financially supported by the Agents of Change award from the National Science Foundation's Human and Social Dynamics program (BCS-0826779, BCS0826840) to K.E.J.; awards from the National Institutes of Health - a National Institute of Allergy and Infectious Diseases nonbiodefense EID Research Opportunities Award (1 R01 AI079231-01) to P.D. and M.V. and a Fogarty International Center American Recovery and Reinvestment Act award (3R01TW005869-06S1) to K.J.O.; and funds from the Ecosystem Services for Poverty Alleviation (ESPA) pro- gram (NE-J001570-1) to K.E.J. and the USAID Emerging Pandemic Threats PREDICT program to L.B., K.E.J., P.D., and K.J.O. The ESPA program is funded by the Department for International Development, the Economic and Social Research Council, and the Natural Environment Research Council. The contents of this work are the responsibility of the authors and do not necessarily reflect the views of USAID or the United States government. We would also like to thank D. Redding and anonymous reviewers for comments on early versions of the manuscript and T. Bogich, K. Safi, and C. Zambrana-Torrelio for assistance.

\section{Literature Cited}

Anderson, R. M., R. M. May, K. Joysey, D. Mollison, G. R. Conway, R. Cartwell, H. V. Thompson, and B. Dixon. 1986. The invasion, persistence and spread of infectious diseases within animal and plant communities. Philosophical Transactions of the Roval Societv B: Biological Sciences 314:533-570.

Anthony, S. J., J. H. Epstein, K. A. Murray, I. Navarrete-Macias, C. M. Zambrana-Torrelio, A. Solovyov, R. Ojeda-Flores, et al. 2013. A strategy to estimate unknown viral diversity in mammals. mBio 4:e00598-13.

Apweiler, R., A. Bairoch, C. H. Wu, W. C. Barker, B. Boeckmann, S. Ferro, E. Gasteiger, et al. 2004. UniProt: the universal protein knowledgebase. Nucleic Acids Research 32:D115-D119.

Balk, D., and G. Yetman. 2005. The global distribution of population: Gridded Population of the World, version 3 (GPWv3). Center for International Earth Science Information Network (CIESIN), New York.

Bausch, D. G., and L. Schwarz. 2014. Outbreak of Ebola virus disease in Guinea: where ecology meets economy. PLoS Neglected Tropical Diseases 8:e3056.

Besag, J. 1974. Spatial interaction and the statistical analysis of lattice systems. Journal of the Royal Statistical Society B: Statistical Methodology 36:192-236.

Bivand, R., and G. Piras. 2015. Comparing implementations of estimation methods for spatial econometrics. Journal of Statistical Software 63:1-36.

Blackburn, T. M., P. Pysek, S. Bacher, J. T. Carlton, R. P. Duncan, V. Jarosik, J. R. Wilson, and D. M. Richardson. 2011. A proposed unified framework for biological invasions. Trends in Ecology and Evolution 26:333-339.

Brierley, L., M. J. Vonhof, K. J. Olival, P. Daszak, and K. E. Jones. 2016. Data from: Quantifying global drivers of zoonotic bat viruses: a process-based perspective. American Naturalist, Dryad Digital Repository, http://dx.doi.org/10.5061/dryad.ds2nj.

Calisher, C. H., J. E. Childs, H. E. Field, K. V. Holmes, and T. Schountz. 2006. Bats: important reservoir hosts of emerging viruses. Clinical Microbiology Reviews 19:531-545.

Chan, E. H., T. F. Brewer, L. C. Madoff, M. P. Pollack, A. L. Sonricker, M. Keller, C. C. Freifeld, M. Blench, A. Mawudeku, and J. S. Brownstein. 2010. Global capacity for emerging infectious disease detection. Proceedings of the National Academv of Sciences of the USA 107:21701-21706.

Chen, L., B. Liu, J. Yang, and Q. Jin. 2014. DBatVir: the database of bat-associated viruses. Database, doi:10.1093/database/bau021. 
Chua, K. B., W. J. Bellini, and P. A. Rota. 2003. Nipah virus outbreak in Malaysia. Journal of Clinical Virology 26:265-275.

Cooper, N., R. Griffin, M. Franz, M. Omotayo, and C. L. Nunn. 2012. Phylogenetic host specificity and understanding parasite sharing in primates. Ecology Letters 15:1370-1377.

Daszak, P. 2009. A call for "smart surveillance": a lesson learned from H1N1. EcoHealth 6:1-2.

Daszak, P., A. A. Cunningham, and A. D. Hyatt. 2000. Emerging infectious diseases of wildlife - threats to biodiversity and human health. Science 287:443-449.

Daszak, P., C. Zambrana-Torrelio, T. L. Bogich, M. Fernandez, J. H. Epstein, K. A. Murray, and H. Hamilton. 2012. Interdisciplinary approaches to understanding disease emergence: the past, present, and future drivers of Nipah virus emergence. Proceedings of the National Academy of Sciences of the USA 110:3681-3688.

Dormann, C. F., J. M. McPherson, M. B. Araújo, R. Bivand, J. Bolliger, G. Carl, R. G. Davies, et al. 2007. Methods to account for spatial autocorrelation in the analysis of species distributional data: a review. Ecography 30:609-628.

Dunn, R. R., T. J. Davies, N. C. Harris, and M. C. Gavin. 2010. Global drivers of human pathogen richness and prevalence. Proceedings of the Roval Society B: Biological Sciences 277:2587-2595.

Epstein, J. H., K. J. Olival, J. R.. Pulliam, C. Smith, J. Westrum, T. Hughes, A. P. Dobson, et al. 2009. Pteropus vampyrus, a hunted migratory species with a multinational home-range and a need for regional management. Journal of Applied Ecology 46:991-1002.

ESRI. 2006. ArcGIS Desktop 9.2. ESRI (Environmental Systems Resource Institute). Redlands, CA. http://www.esri.com/software/arcgis.

Farrer, R. A., L. A. Weinert, J. Bielby, T. W. J. Garner, F. Balloux, F. Clare, J. Bosch, et al. 2011. Multiple emergences of genetically diverse amphibian-infecting chytrids include a globalized hypervirulent recombinant lineage. Proceedings of the National Academy of Sciences of the USA 108:18732-18736.

Fritz, S. A., and A. Purvis. 2010. Phylogenetic diversity does not capture body size variation at risk in the world's mammals. Proceedings of the Roval Society B: Biological Sciences 277:2435-2441.

Funk, S., T. L. Bogich, K. E. Jones, A. M. Kilpatrick, and P. Daszak. 2013. Quantifying trends in disease impact to produce a consistent and reproducible definition of an emerging infectious disease. PLoS ONE 8:e69951.

Guan, Y., B. J. Zheng, Y. Q. He, X. L. Liu, Z. X. Zhuang, C. L. Cheung, S. W. Luo, et al. 2003. Isolation and characterization of viruses related to the SARS coronavirus from animals in southern China. Science 302:276-278.

Guernier, V., M. E. Hochberg, and J. F. Guégan. 2004. Ecology drives the worldwide distribution of human diseases. PLoS Biology 2 e141.

Halpin, K., A. D. Hyatt, R. K. Plowright, J. H. Epstein, P. Daszak, H. E. Field, L.-F. Wang, P. W. Daniels, and Henipavirus Ecology Research Group. 2007. Emerging viruses: coming in on a wrinkled wing and a prayer. Clinical Infectious Diseases 44:711-717.

Harris, N. C., and R. R. Dunn. 2010. Using host associations to predict spatial patterns in the species richness of the parasites of North American carnivores. Ecology Letters 13:1411-1418.

Hijmans, R. J., S. E. Cameron, J. L. Parra, P. G. Jones, and A. Jarvis. 2005 . Very high resolution interpolated climate surfaces for global land areas. International Journal of Climatology 25:1965-1978.

Hopkins, M. E., and C. L. Nunn. 2007. A global gap analysis of infectious agents in wild primates. Diversity and Distributions 13: 561-572.
Hulme, P. E., S. Bacher, M. Kenis, S. Klotz, I. Kühn, D. Minchin, W. Nentwig, et al. 2008. Grasping at the routes of biological invasions: a framework for integrating pathways into policy. Journal of Applied Ecology 45:403-414.

Jones, K. E., N. G. Patel, M. A. Levy, A. Storeygard, D. Balk, J. L. Gittleman, and P. Daszak. 2008. Global trends in emerging infectious diseases. Nature 451:990-993.

Kamins, A. O., O. Restif, Y. Ntiamoa-Baidu, R. Suu-Ire, D. T. S. Hayman, A. A. Cunningham, J. L. N. Wood, and J. M. Rowcliffe. 2011. Uncovering the fruit bat bushmeat commodity chain and the true extent of fruit bat hunting in Ghana, West Africa. Biological Conservation 144:3000-3008.

Karesh, W. B., A. Dobson, J. O. Lloyd-Smith, J. Lubroth, M. A. Dixon, M. Bennett, S. Aldrich, et al. 2012. Ecology of zoonoses: natural and unnatural histories. Lancet 380:1936-1945.

King, A. M., E. Lefkowitz, M. J. Adams, and E. B. Carstens. 2011. Virus taxonomy: ninth report of the International Committee on Taxonomy of Viruses. Elsevier, San Diego, CA.

Leroy, E. M., A. Epelboin, V. Mondonge, X. Pourrut, J. P. Gonzalez, J. J. Muyembe-Tamfum, and P. Formenty. 2009. Human Ebola outbreak resulting from direct exposure to fruit bats in Luebo, Democratic Republic of Congo, 2007. Vector-Borne and Zoonotic Diseases 9:723-728.

Li, W., Z. Shi, M. Yu, W. Ren, C. Smith, J. H. Epstein, H. Wang, et al. 2005. Bats are natural reservoirs of SARS-like coronaviruses. $\underline{\text { Sci- }}$ ence 310:676-679.

Lloyd-Smith, J. O., D. George, K. M. Pepin, V. E. Pitzer, J. R. C. Pulliam, A. P. Dobson, P. J. Hudson, and B. T. Grenfell. 2009. Epidemic dynamics at the human-animal interface. Science 326: 1362-1367.

Luis, A. D., D. T. S. Hayman, T. J. O’Shea, P. M. Cryan, A. T. Gilbert, J. R. C. Pulliam, J. N. Mills, et al. 2013. A comparison of bats and rodents as reservoirs of zoonotic viruses: are bats special? Proceedings of the Roval Societv B: Biological Sciences 280:20122753.

Memish, Z. A., N. Mishra, K. J. Olival, S. F. Fagbo, V. Kapoor, J. H. Epstein, R. AlHakeem, et al. 2013. Middle East Respiratory Syndrome coronavirus in bats, Saudi Arabia. Emerging Infectious Diseases 19:1819-1823.

Mickleburgh, S., K. Waylen, and P. Racey. 2009. Bats as bushmeat: a global review. Orvx 43:217-234.

Miller-Butterworth, C. M., D. S. Jacobs, and E. H. Harley. 2003. Strong population substructure is correlated with morphology and ecology in a migratory bat. Nature 424:187-191.

Mollentze, N., R. Biek, and D. G. Streicker. 2014. The role of viral evolution in rabies host shifts and emergence. Current Opinion in Virology 8:68-72.

Morse, S. S. 1995. Factors in the emergence of infectious diseases. Emerging Infectious Diseases 1:7-15.

Morse, S. S., J. A. Mazet, M. Woolhouse, C. R. Parrish, D. Carroll, W. B. Karesh, C. Zambrana-Torrelio, W. I. Lipkin, and P. Daszak. 2012. Prediction and prevention of the next pandemic zoonosis. Lancet 380:1956-1965.

Nahar, N., R. Sultana, E. S. Gurley, M. J. Hossain, and S. P. Luby. 2010. Date palm sap collection: exploring opportunities to prevent Nipah transmission. EcoHealth 7:196-203.

Negredo, A., G. Palacios, S. Vázquez-Morón, F. González, H. Dopazo, F. Molero, J. Juste, et al. 2011. Discovery of an ebolavirus-like filovirus in Europe. PLoS Pathogens 7:e1002304.

Olival, K. J., J. H. Epstein, L.-F. Wang, and H. E. Field. 2012. Are bats exceptional viral reservoirs? Pages 195-212 in A. A. Aguirre, 
R. S. Ostfeld, and P. Daszak, eds. New directions in conservation medicine: applied cases of ecological health. Oxford University Press, Oxford.

Patz, J. A., P. Daszak, G. M. Tabor, A. A. Aguirre, M. Pearl, J. Epstein, N. D. Wolfe, et al. 2004. Unhealthy landscapes: policy recommendations on land use change and infectious disease emergence. Environmental Health Perspectives 112:1092-1098.

Pedersen, A. B., and T. J. Davies. 2009. Cross-species pathogen transmission and disease emergence in primates. EcoHealth 6:496-508.

Pigott, D. M., N. Golding, A. Mylne, Z. Huang, A. J. Henry, D. J. Weiss, O. J. Brady, et al. 2014. Mapping the zoonotic niche of Ebola virus disease in Africa. eLife 3:e04395.

Plowright, R. K., P. Foley, H. E. Field, A. P. Dobson, J. E. Foley, P. Eby, and P. Daszak. 2011. Urban habituation, ecological connectivity and epidemic dampening: the emergence of Hendra virus from flying foxes (Pteropus spp.). Proceedings of the Roval Society B: Biological Sciences 278:3703-3712.

Plowright, R. K., S. H. Sokolow, M. E. Gorman, P. Daszak, and J. E. Foley. 2008. Causal inference in disease ecology: investigating ecological drivers of disease emergence. Frontiers in Ecology and the Environment 6:420-429.

Pulliam, J. R. C. 2008. Viral host jumps: moving toward a predictive framework. EcoHealth 5:80-91.

Pulliam, J. R. C., J. H. Epstein, J. Dushoff, S. A. Rahman, M. Bunning, A. A. Jamaluddin, A. D. Hyatt, et al. 2012. Agricultural intensification, priming for persistence and the emergence of Nipah virus: a lethal bat-borne zoonosis. Journal of the Roval Society Interface 9:89-101.

Rahman, S. A., S. S. Hassan, K. J. Olival, M. Mohamed, L.-Y. Chang, L. Hassan, N. M. Saad, et al. 2010. Characterization of Nipah virus from naturally infected Pteropus vampyrus bats, Malaysia. Emerging Infectious Diseases 16:1990-1993.

Ramankutty, N. 2010. Global cropland and pasture data from 17002007. The LUGE (Land Use and the Global Environment) lab. http://www.earthstat.org/.

R Development Core Team. 2011. R: a language and environment for statistical computing. R Foundation for Statistical Computing, Vienna. http://www.R-project.org.

Reusken, C. B. E. M., B. L. Haagmans, M. A. Müller, C. Gutierrez, G.-J. Godeke, B. Meyer, D. Muth, et al. 2013. Middle East respiratory syndrome coronavirus neutralising serum antibodies in dromedary camels: a comparative serological study. Lancet Infectious Diseases 13:859-866.

Rossiter, S. J., G. Jones, R. D. Ransome, and E. M. Barratt. 2000. Genetic variation and population structure in the endangered greater horseshoe bat Rhinolophus ferrumequinum. Molecular Ecology 9:1131-1135.

Sandvik, B. 2008. Thematic Mapping: World Borders Dataset. Available under a Creative Commons Attribution-Share Alike License. http://thematicmapping.org/downloads/world_borders.php.

Smolinski, M. S., M. A. Hamburg, and J. Lederberg. 2003. Microbial threats to health: emergence, detection, and response. National Academies Press, Washington, DC.

Streicker, D. G., A. S. Turmelle, M. J. Vonhof, I. V. Kuzmin, G. F McCracken, and C. E. Rupprecht. 2010. Host phylogeny con- strains cross-species emergence and establishment of rabies virus in bats. Science 329:676-679.

Taylor, L. H., S. M. Latham, and M. E. J. Woolhouse. 2001. Risk factors for human disease emergence. Philosophical Transactions of the Roval Societv B: Biological Sciences 356:983-989.

Tong, S., Y. Li, P. Rivailler, C. Conrardy, D. A. A. Castillo, L.-M. Chen, S. Recuenco, et al. 2012. A distinct lineage of influenza A virus from bats. Proceedings of the National Academv of Sciences of the USA 109:4269-4274.

Towner, J. S., X. Pourrut, C. G. Albariño, C. N. Nkogue, B. H. Bird, G. Grard, T. G. Ksiazek, J.-P. Gonzalez, S. T. Nichol, and E. M. Leroy. 2007. Marburg virus infection detected in a common African bat. PLoS ONE 2:e764.

Turmelle, A. S., and K. J. Olival. 2009. Correlates of viral richness in bats (order Chiroptera). EcoHealth 6:522-539.

USAID. 2009. USAID launches Emerging Pandemic Threats program. Press release, October 21, 2009. United States Agency for International Development, Washington, DC.

Viana, M., R. Mancy, R. Biek, S. Cleaveland, P. C. Cross, J. O. LloydSmith, and D. T. Haydon. 2014. Assembling evidence for identifying reservoirs of infection. Trends in Ecology and Evolution 29:270-279.

Webb, S. D., M. J. Keeling, and M. Boots. 2007. Host-parasite interactions between the local and the mean-field: how and when does spatial population structure matter? Journal of Theoretical Biology 249:140-152.

Wilson, D. E., and D. A. Reeder. 2005. Mammal species of the world: a taxonomic and geographic reference. 3rd ed. Johns Hopkins University Press, Baltimore.

Wint, W., and T. Robinson. 2007. Gridded Livestock of the World 2007. FAO, Rome.

Wolfe, N. D., C. P. Dunavan, and J. Diamond. 2007. Origins of major human infectious diseases. Nature 447:279-283.

Wolfe, N. D., M. N. Eitel, J. Gockowski, P. K. Muchaal, C. Nolte, A. T. Prosser, J. N. Torimiro, S. F. Weise, and D. S. Burkeet. 2000. Deforestation, hunting and the ecology of microbial emergence. Global Change and Human Health 1:10-25.

Wood, J. L. N., M. Leach, L. Waldman, H. MacGregor, A. R. Fooks, K. E. Jones, O. Restif, et al. 2012. A framework for the study of zoonotic disease emergence and its drivers: spillover of bat pathogens as a case study. Philosophical Transactions of the Roval Societv B: Biological Sciences 367:2881-2892.

Woolhouse, M. E. J., K. Adair, and L. Brierley. 2013. RNA viruses: a case study of the biology of emerging infectious diseases. Microbiology Spectrum 1:OH-0001-2012.

Woolhouse, M. E. J., and S. Gowtage-Sequeria. 2005. Host range and emerging and reemerging pathogens. Emerging Infectious Diseases 11:1842-1847.

World Bank Group. 2010. The World Bank databank: world development indicators and global development finance. http://databank .worldbank.org/data/home.aspx. 